\title{
Does node stability underlie the verbal transformation effect? A test of node structure theory
}

\author{
LISA CONTOS SHOAF and MARK A. PITT \\ Ohio State University, Columbus, Ohio
}

\begin{abstract}
Continuous repetition of a word causes listeners to hear the word transform into other utterances, an illusion known as the verbal transformation effect. Node structure theory (MacKay, 1987) provides a useful framework for understanding the illusion, positing that the transformations listeners report are a function of the stability of the node that represents the repeating stimulus. In Experiment 1, the accuracy of this account was investigated, using stimuli that varied from words to phonotactically illegal pseudowords. Experiments 2 and 3 replicated and generalized the findings of Experiment 1, which support a conceptualization of node stability slightly different from that embodied in node structure theory. A new method of measuring lexical influences in the verbal transformation effect is also introduced.
\end{abstract}

Illusions arise when the perceptual system breaks down. Researchers study illusions to enhance their understanding of the otherwise hidden mechanisms responsible for accurate perception (Warren, 1983). Studying verbal illusions, therefore, may reveal something about the functioning of the language system. One such illusion is the verbal transformation effect, first reported by Warren and Gregory (1958). They found that listeners who were presented with a continuously repeating word or phrase began to perceive transformations - that is, changes in the repeating stimulus, relative to what the listener had perceived on preceding repetitions of the stimulus. Transformations ranged from one-phoneme alterations to drastic phonologicaldistortions. For example, when presented with the word truce, participants reported hearing phonetically similar transformations, such as struce and truth, and the pseudoword struth, as well as dissimilar transformations, such as Esther.

What causes verbal transformations? The most detailed accounts of the verbal transformation effect to date, which will be evaluated in this paper, are derived from a broad theory of speech perception and production known as node structure theory (MacKay, 1987; MacKay, Wulf, Yin, \& Abrams, 1993). Node structure theory is instantiated as a localist network model, with nodes (representational units) hierarchically organized into three levels: the muscle move-

This work was supported by Research Grant R29 DC01774 from the National Institute on Deafness and Other Communication Disorders, National Institutes of Health. We thank Donald MacKay for his very helpful review of this manuscript and for his willingness to personally respond to our questions. We also thank Lyn Canterbury, Kalyani Subramaniam, David Barthel, and Christina Ingler for help in testing participants and scoring data. Correspondence concerning this article should be addressed to L. C. Shoaf or M. A. Pitt, Department of Psychology, Ohio State University, 1885 Neil Avenue, Columbus, OH 43210 (e-mail: contos.1@osu.edu or pitt.2@osu.edu). ment level, the phonologicallevel, and the sentential level. The muscle movement level contains nodes specifically for the production of speech. Nodes at the phonological level represent sublexical linguistic units, such as syllables, subsyllabic segments (e.g., onset or rhyme), and phonetic features. The sentential level contains lexical nodes for words and phrases.

Both perception and production operate on the same nodes at the phonological and sentential levels. In perception, speech input first primes nodes at the feature level. Priming produces increased subthreshold activity (but not activation, as the term is generally used) in a node. It spreads in parallel across nodes, with its strength being positively related to how well it matches the input. In the same fashion, priming spreads to the phonological nodes and, finally, to the lexical nodes. At each level, priming strength is a function of the match to the input from the preceding level. Although several nodes may be primed at once, only the node that is primed to a higher degree than all the others becomes activated.

How pseudowords are recognized and new lexical entries are formed is also described by node structure theory. Connections from nodes in the phonological system converge to form temporary lexical nodes that represent pseudowords. These connections are initially weak, and without frequent activation, these newly formed and fragile connections will decay. Repeated use of the pseudoword will eventually "commit" the node to permanent status, at which point it will become a new entry in the lexicon (MacKay, Burke, \& Stewart, 1998; Yin \& MacKay, 1992).

Unique among models of speech perception, node structure theory provides a detailed account of the dynamics of representational units during repeated stimulation, which allows one to make precise predictions regarding the verbal transformation effect. In node structure theory, verbal transformations occur because of node satiation, which is 
a drop in the maximum attainable priming level of a node (MacKay et al., 1993). Nodes at the lexical level or at the phonological level can become satiated. Satiation occurs because of repeated activation (not merely priming) of a node from continual repetition of the same utterance (e.g., the word cast). As satiation increases, the node's priming level drops, eventually falling below that of a competitor node (e.g., the lexical node for fast) that is only moderately primed by the speech input, because of one or more mismatching phonological segments. Because the most primed node becomes activated in node structure theory, the competitor node (e.g., the lexical node for fast), which now has the highest priming level, will then become activated, resulting in the perception of a transformation (i.e., the word fast) by the listener.

As the preceding description suggests, the amount of priming a node receives greatly influences the transformations perceived (Yin \& MacKay, 1992). A node that is unable to attain a great deal of priming will rarely become activated. One other factor that influences transformations, according to node structure theory, is neighborhood density (Luce, Pisoni, \& Goldinger, 1990), which is the number of lexical entries (i.e., neighbors) that are phonetically similar to the repeating stimulus. The more neighbors there are, the greater the number of possible competitors there are to become activated, resulting in a greater number and a wider range of transformations. Yin and MacKay (1992) have reported data that confirm this prediction.

At the heart of node structure theory's account of the verbal transformation effect is the concept of lexical node stability, which refers to the extent to which a node remains activated over its competitors. The more stable a node is, the less frequently it will lose out to other lexical competitors becoming activated. Stability is a function of a number of variables, such as the amount of priming transmitted to the lexical node from phonological nodes (i.e., acoustic-phonetic fit), neighborhood density, and strength of its connections, which is directly related to frequency of use. Thus, node stability should be inversely related to transformation frequency. In particular, nodes for words, which have strong and well-formed representations, should be more stable than nodes for pseudowords. Data from a few experiments partially support this prediction. Warren (1961b; see also Warren, 1961a; Warren \& Warren, 1966) recorded the number of transformations that listeners reported when hearing words and pseudowords repeat. He found that pseudowords elicited more transformations than did words, suggesting that nodes for pseudowords are less stable than nodes for words. In an analysis of the specific transformations (i.e., forms) that listeners reported, Natsoulas (1965) found a similar asymmetry, with pseudowords eliciting more forms than did words. Natsoulas also discussed the verbal transformation effect in terms of satiation and perceptual stability.

Construction of an accurate model of word perception requires specifying the operation of representational units. At present, knowledge about their operation is lacking, which is why decisions on how to implement them in com- putational models, such as TRACE (McClelland \& Elman, 1986) and Merge (Norris, McQueen, \& Cutler, 2000), must be guided by intuitions and indirect evidence. The hypothesized mechanisms of satiation and recovery, as embodied in node structure theory, begin to fill this gap by describing how reactivation of representational units might occur. The verbal transformation effect is well suited for testing the validity of this proposal, because the frequent perception of transformations provides a means of measuring node stability and, thus, linking it to one or more of these mechanisms. The following experiments were undertaken to explore this idea.

\section{EXPERIMENT 1}

MacKay et al. (1993, pp.628-629) conjectured that according to node structure theory, transformation frequency should increase as an utterance becomes less wordlike. Specifically, they hypothesized that words (e.g., skunk) should yield fewer transformations than do phonotactically permissible pseudowords (e.g., swunk). These legal pseudowords should, in turn, yield fewer transformations than illegal pseudowords (e.g., srunk), which contain strings of phonemes that are phonotactically impermissible in English (e.g., $s r$ cannot occur at the beginning of a word). This prediction is motivated by a consideration of node stability across the three conditions. According to node structure theory, phonotactically legal pseudowords require the formation of a new lexical node (e.g., a lexical node for swunk). The weak connection strength to this newly formed node makes it less stable and, thus, more likely to lose out to stronger competitors, resulting in more transformations. Phonotactically illegal pseudowords, which require the formation of new nodes at both the lexical level (e.g., a node for srunk) and the phonological level (e.g., a node for $s r$ ), yield an even weaker, more unstable representation and will be even more likely to lose out to stronger competitors. Experiment 1 tested this prediction.

An additional aim of Experiment 1 was to demonstrate lexical effects in the verbal transformation effect more definitively than have past studies. Other variables besides lexical status have varied between words and pseudowords in these experiments. For example, in the groundbreaking experiments on the verbal transformation effect (e.g., Warren, 1961a; Warren \& Warren, 1966), few pairs of words and pseudowords were used, and the stimuli covaried along dimensions other than lexical status (e.g., number of stimulus repetitions or acoustic-phonetic characteristics), making it difficult to attribute differences solely to lexical status. The most well-controlled study (Natsoulas, 1965) found no differences as a function of lexical status when the number of transformations was measured but did find a difference when the number of unique forms was tallied. However, instead of presenting the stimulus continuously for a specified number of repetitions, Natsoulas inserted 15 -sec pauses between every 60 repetitions. This change in procedure complicates comparison of his data with other findings. 
In addition to using a larger set of stimuli and controlling for acoustic differences across stimulus conditions as much as possible, node stability was assessed by using two measures of the verbal transformation effect, the number of transformations reported and the number of unique forms reported. Although MacKay et al. (1993) do not explicitly discuss predictions of node structure theory with the latter measure, it seemed reasonable to suppose that the theory would predict that the number of forms listeners report would increase as stability decreased, because the fragile representation of a pseudoword has the potential to lose out to many strong competitors, whereas the comparatively much stronger representation of a word will lose out to far fewer competitors, resulting in fewer unique transformations.

\section{Method}

Participants. Eighteen undergraduates from an introductory psychology class participated for credit. All were native English speakers and reported normal hearing.

Stimuli. There were three conditions: a word condition, a legal pseudoword condition, and an illegal pseudoword condition. The stimuli in the illegal pseudoword condition contained an initial consonant cluster that violated rules of English phonotactics (e.g., /tl/, /sr/). We attempted to match stimuli phonetically across conditions as closely as possible so that any differences found between the three conditions would be attributed to differences in lexical status, rather than to other properties of the stimuli (e.g., acoustic-phonetic variation). For three of the six triplets of stimuli, the items varied only in the second consonant of the cluster (e.g., skunk, swunk, srunk). In the other three, such strict control could not be maintained, because of language constraints. As a result, the initial consonant also varied between words and pseudowords, but not between the legal and the illegal pseudowords (e.g., blond, trond, tlond). These three sets of triplets produced the same patterns of data, indicating that this additional variation had no measurable effect on performance.

The number of phonemes (mean length of 4.3), as well as the syllable structure of the stimuli (CCVC or CCVCC), was held constant within each triplet. Mean frequency for words was 18.5. Mean neighborhood density (defined as the number of phonologically similar words that differed from the stimulus only by a single substitution, insertion, or deletion) was 6.83 for words and 6.50 for legal pseudowords. Density for illegal pseudowords was understandably less (2.10; the presence of an illegal cluster will, by definition, constrain the size of the neighborhood). Not surprisingly, mean neighborhood frequency patterned similarly across conditions (18.3 for words, 25.5 for pseudowords, and 13.7 for illegal pseudowords). Because MacKay et al. (1993) had stated that frequency and density affected node stability, we held these factors constant as best as possible, to neutralize their impact.

L.C.S. practiced pronouncing the illegal pseudowords extensively before recording the tokens, and their intelligibility was assessed in a pilot study (15 listeners) to ensure that the illegal consonant cluster was heard as intended (the phonemes of the illegal cluster were spelled correctly $92 \%$ of the time). The stimuli are listed in the Appendix.

All the stimuli were recorded onto digital audio tape at a $48-\mathrm{kHz}$ sampling rate and were digitally transferred to the hard disk of a PC (downsampled to $16 \mathrm{kHz}$, low-pass filtered at $7.6 \mathrm{kHz}$ ), where they were edited and saved as individual sound files. Mean duration was $680 \mathrm{msec}$ for words, $741 \mathrm{msec}$ for legal pseudowords, and $766 \mathrm{msec}$ for illegal pseudowords.

Apparatus. Stimulus presentation was controlled by a microcomputer. The stimuli were presented binaurally over headphones at a listening level deemed comfortable by the experimenter. Trans- formations were reported into a microphone and recorded onto cassette tape.

Procedure. The participants were tested up to two at a time in separate sound-attenuated booths. They were told that they would hear a word or a nonsense word repeat through the headphones and that they were to listen carefully for any transformations in the repeating stimulus. A transformation was defined as a change in the repeating utterance to another word or to a nonsense word. Transformations were reported into a microphone placed in front of the seated participant. The participants were informed that the transformations could be subtle or very noticeable and that they were to report any and all transformations, words and nonsense words alike. The participants were assured that there were no correct or incorrect responses and that if they did not hear any transformations, they were to say nothing.

The experiment began with two practice trials consisting of the word grace and the pseudoword blit. In the test phase, the participants received one of three possible randomly ordered lists of stimuli. Each list contained two stimuli from each of the three conditions, for a total of six test trials. Each trial consisted of 350 repetitions, with a $250-\mathrm{msec}$ interstimulus interval (ISI) between repetitions. Lengthy breaks were offered between trials. The experiment lasted $45 \mathrm{~min}$.

\section{Results}

Number of transformations. The data were scored by summing the total number of transformations reported for each item by each participant. The data were then averaged across participants. Mean numbers of transformations are shown in the left half of Table 1. Overall, legal pseudowords yielded essentially the same number of transformations as did words. Counter to node structure theory, illegal pseudowords yielded 3.70 fewer transformations than did legal pseudowords. The one-way analysis of variance (ANOVA) across conditions was not reliable $\left[F(2,34)=2.04, M S_{\mathrm{e}}=77.28 ; p\right.$ values exceeded the .05 level, unless otherwise stated].

Because there were only six items in each condition, inferential statistics were not performed on the item data. Instead, item triplets (e.g., skunk, swunk, srunk) were compared across conditions, which yielded similarly equivocal findings. Legal pseudowords produced more transformations than did words in three out of six sets of items. Legal pseudowords also yielded more transformations than did illegal pseudowords in four out of six sets, and words yielded more transformations than did illegal pseudowords in four out of six sets.

Number of forms. The mean number of forms produced in each of the three conditions was calculated in the same manner as the number of transformations, and the results are shown in the right side of Table 1 . The results

Table 1

Mean Number of Transformations and Forms Reported as a Function of Stimulus Type for Experiment 1 (With Standard Deviations)

\begin{tabular}{lccccc}
\hline & \multicolumn{2}{c}{ Transformations } & & \multicolumn{2}{c}{ Forms } \\
\cline { 2 - 3 } \cline { 5 - 6 } Stimulus Type & $M$ & $S D$ & & $M$ & $S D$ \\
\hline Word & 34.89 & 19.30 & & 4.67 & 1.39 \\
Legal pseudoword & 35.11 & 20.70 & & 7.06 & 1.84 \\
Illegal pseudoword & 31.41 & 14.86 & & 6.83 & 2.44 \\
\hline
\end{tabular}


only partially supported the predictions of node structure theory. The one-way ANOVA was reliable $[F(2,34)=$ $\left.14.16, M S_{\mathrm{e}}=2.21\right]$. Although legal pseudowords yielded 2.39 more forms than did words, illegal pseudowords did not yield more forms than did legal pseudowords. Instead, the two means were nearly identical. Paired comparisons with a Bonferroni adjustment showed that this effect was due solely to the fact that the number of forms in the word condition differed reliably from those in the legal pseudoword condition $[t(1,17)=5.34]$ and the illegal pseudoword condition $[t(1,17)=3.87]$. The item data yielded a similarly equivocal outcome. For five of six triplets, more forms were reported to legal pseudowords than to words, but for four of six triplets, more forms were reported to legal pseudowords than to illegal pseudowords.

The null results found in the transformation analysis and the mixed results found in the form analysis provide weak support for node stability as instantiated in node structure theory. However, because few differences were found across conditions, the data could also suggest that these dependent measures are poor measures of node stability. The latter explanation may be particularly true because, in the transformation and form analyses, each participant's reports are lumped together into one composite value, possibly obscuring differences in the perceptual experience of the listener. That the data are more informative than was revealed by the two global measures is suggested by the strength of the correlations between the number of transformations and the number of forms. Whereas robust positive correlations were found in the legal and illegal pseudoword conditions (.45 and .23, respectively), none was obtained in the word condition $(-.03)$, suggesting something different was going on in the latter condition.

To pursue this hunch, a new analysis was developed that was intended to measure node stability more directly. Transformations back to the veridical stimulus (i.e., the intended word) were tallied independently of nonveridical transformations. Our reasoning was that the greater the number of veridical transformations that listeners report, the more stable the percept. That is, stability should be a direct function of the frequency with which an utterance transforms back into the intended word. A large number of nonveridical transformations reflects far less perceptual stability.
Form subanalysis. All transformations were classified as a function of whether they were veridical (i.e., matched the spoken stimulus) or nonveridical. When the latter was reported, the transformation was further classified as either a word or a pseudoword. The data are shown in Table 2. Although the data are discussed in terms of the proportion of transformations, statistical analyses on the frequency counts yielded the same outcome.

When the stimulus was a word, it transformed back into the veridical stimulus more often than when it was a pseudoword. Interestingly, illegal pseudowords did so very infrequently, only $10 \%$ of the time. The one-way ANOVA across conditions was reliable $\left[F(2,34)=31.76, M S_{\mathrm{e}}=0.01\right]$. Planned comparisons showed that all three veridical means differed reliably from each other. The nonveridical transformations revealed what listeners heard when they did not hear the veridical form. Across all three stimulus types, transformations into other words fell into the small range of .23-.30, with no differences between means being reliable $\left[F(2,34)<1, M S_{\mathrm{e}}=0.02\right]$.

What differed significantly across the stimulus categories was the number of nonveridical pseudoword transformations. As the stimulus became less wordlike, the number of nonveridical pseudoword transformations increased $\left[F(2,34)=10.34, M S_{\mathrm{e}}=0.03\right]$. Illegal pseudowords yielded the largest proportion of nonveridical pseudoword transformations (.60), and words yielded the smallest (.36). Paired comparisons showed that all the means differed reliably, except those between the legal and the illegal pseudowords.

\section{Discussion}

The results of Experiment 1 supported the idea of node stability, but not in the way predicted by MacKay et al. (1993). In the overall transformation analysis, only small differences (none statistically reliable) were found among the three conditions. In the form analysis, significant differences were found between words and both types of pseudowords, but not between legal and illegal pseudowords. These results replicated closely those of Natsoulas (1965), who found no reliable differences between words and pseudowords when they were compared on the number of transformations but found consistent differences when they were compared on the number of forms. These

Table 2

Mean Proportions of Veridical and Nonveridical Transformations Reported as a Function of Stimulus Type in Experiment 1 (With Mean Numbers of Transformations)

\begin{tabular}{|c|c|c|c|c|c|c|}
\hline \multirow[b]{3}{*}{ Transformation Type } & \multicolumn{6}{|c|}{ Stimulus Type } \\
\hline & \multicolumn{2}{|c|}{ Word } & \multicolumn{2}{|c|}{ Legal Pseudoword } & \multicolumn{2}{|c|}{ Illegal Pseudoword } \\
\hline & Prop. & No. & Prop. & No. & Prop. & No. \\
\hline Veridical transformations & .39 & 14.17 & .25 & 10.39 & .10 & 3.97 \\
\hline Nonveridical transformations & .61 & 20.72 & .75 & 25.00 & .90 & 27.44 \\
\hline Words & .25 & 7.89 & .23 & 6.97 & .30 & 8.58 \\
\hline Pseudowords & .36 & 12.83 & .52 & 18.03 & .60 & 18.86 \\
\hline
\end{tabular}

Note-Standard deviations ranged from .13 to .28 . 
data also suggest that in past work in which differences between words and pseudowords on the measure of number of transformations were reported, the differences may have been due to variables other than lexical status.

The form subanalysis elaborated on the overall form analysis by revealing that node stability did vary as a function of lexical status. In the process, a new characteristic of the verbal transformation effect was identified. Words do, in fact, yield more stable percepts than do pseudowords (legal and illegal), not so much because they yield fewer transformations, but because the stimuli transform back into the veridical form more often. Furthermore, the less wordlike the utterance is (legal vs. illegal pseudowords), the less often it transforms back into the veridical stimulus. This pattern of results, although found with a different measure, is what node structure theory predicts.

Inspection of the nonveridical transformations further revealed how reports across the three conditions differed. The proportion of nonveridical word transformations was relatively the same, ranging from .23 to .30 . On the other hand, the proportion of nonveridical pseudoword transformations varied greatly as a function of lexical status, with words producing the fewest transformations (.36) and legal and illegal pseudowords producing the most and similar amounts (.52 and .60, respectively). It is difficult to derive predictions from node structure theory on the basis of these additional findings, although they do suggest that lexical influences manifest themselves in other ways as well.

That participants reported pseudowords as transformations is easily explained by node structure theory as being the result of the formation of new connections. However, what node structure theory does not readily explain is the high frequency with which pseudowords were reported as transformations. One possibility is that the sheer number of possible pseudoword neighbors (i.e., many combinations of sublexical nodes), as opposed to word neighbors (which averaged under 7.0 across all conditions) makes the likelihood that a pseudoword will be reported as a transformation very high. In other words, regardless of a node's stability, once it becomes satiated, any phonetically similar item, word or pseudoword, may become activated. This, along with instructions to the participants that specifically stated that pseudowords were acceptable responses, may help to explain the high proportion of nonveridical pseudoword reports in this experiment and in the two that will follow. 1

It is worth considering whether the drop in veridical reports across conditions could also be due to other factors that vary with lexical status. For example, the rarity of veridical reports in the illegal pseudoword condition could also be due to the unfamiliar pronunciation of pseudowords. Although ease of pronunciation might have increased the size of the effect between the legal and the illegal pseudoword conditions (.15), it is doubtful that this explanation is responsible for all of it. Even the most prolific participants, who appeared to have had little trouble pronouncing the illegal pseudowords given the number of times they were reported (up to 20), showed the same pattern of data. It might also be the case that the participants rejected pseudowords, but not words, as possible percepts. This account seems particularly unlikely, given the ease and frequency with which nonveridical pseudoword transformations were reported (on average, 18 times).

\section{EXPERIMENT 2}

The purpose of Experiment 2 was to replicate and generalize the results of Experiment 1. The form subanalysis was post hoc, applied only after the other two analyses proved only mildly informative. If this analysis is valid and reliable, a similar pattern should be found in a replication. Furthermore, the replication should generalize to other stimuli. To address these issues, the design of Experiment 1 was expanded so we could look at word-pseudoword differences for two-syllable, as well as for one-syllable, stimuli. To keep the design of the experiment within subjects while also keeping the number of trials manageable, the illegal pseudoword condition was dropped.

\section{Method}

Participants. Thirty-one new participants from the same pool as that in Experiment 1 participated.

Stimuli. The monosyllabic stimuli (words and pseudowords) were the words and legal pseudowords used in Experiment 1. Bisyllabic pseudowords were created from bisyllabic words by swapping the order of the two syllables (see the Appendix). Although this method of pseudoword creation was not the same as that used in creating monosyllabic pseudowords, it was thought to be advantageous, because the result was that the words and the pseudowords had acoustically identical syllables, differing only in their order. They were constructed by first making two recordings of each of the twosyllable words and then splicing each at the syllable boundary. We recorded two tokens of each word so that a truly spliced word would be created, rather than just cutting a single word into two syllables only to paste them back together again. The first and last syllables from two different tokens of the word were pasted together in the correct order to create a word stimulus and in the reverse order to create the corresponding pseudoword. Because the medial consonant clusters contained either a stop or a fricative, splicing was relatively easy. The /t/ was moderately aspirated in the pronunciation of "fluster" so that the stop would be heard as /t/ when the two syllables were swapped. Bisyllabic stimuli were an average of 5.8 phonemes in length, with words and pseudowords being identical in duration (overall mean of $757 \mathrm{msec}$ ). Monosyllables were an average of 4.3 phonemes in length. A method of calculating the neighborhood density of multisyllabic words has not been developed, so this analysis was not performed.

A pilot study was run to ensure that the stimuli were heard as intended. Fifteen participants listened to the stimuli over headphones and spelled the words and pseudowords, using any spelling that made sense (i.e., the listeners were instructed not to worry if there was more than one way to spell a particular phoneme). Monosyllabic words and pseudowords were correctly identified $100 \%$ and $98 \%$ of the time, respectively. The bisyllabic stimuli yielded similarly good results. The spliced stimuli were spelled almost as well as the original unspliced tokens (90\% vs. 94\%), although the spliced words were somewhat easier to spell than the spliced pseudowords ( $97 \%$ vs. $83 \%)$.

Procedure. The procedure was identical to that in Experiment 1. The experiment consisted of two practice trials followed by four test trials, in which 1 stimulus was randomly presented from each of the four conditions. Each stimulus was presented for 350 repetitions. For 9 of the 24 stimuli ( 6 of the 12 bisyllables), the ISI was $1,016 \mathrm{msec}$. 
Table 3

Mean Numbers of Transformations and Forms Reported as a Function of Lexical Status and Number of Syllables (With Standard Deviations) in Experiment 2 With Cross-Spliced Stimuli

\begin{tabular}{lccccc}
\hline & \multicolumn{2}{c}{ Transformations } & & \multicolumn{2}{c}{ Forms } \\
\cline { 2 - 3 } \cline { 5 - 6 } Stimulus Type & $M$ & $S D$ & & $M$ & $S D$ \\
\hline One-Syllable & & & & \\
$\quad$ Word & 57.26 & 33.87 & & 7.03 & 3.90 \\
$\quad$ Pseudoword & 58.77 & 34.62 & 7.74 & 4.64 \\
Two-Syllable & & & & \\
$\quad$ Word & 50.94 & 36.48 & 7.10 & 4.11 \\
$\quad$ Pseudoword & 46.29 & 31.81 & 7.61 & 4.94 \\
\hline
\end{tabular}

For the remaining 15 stimuli ( 9 of the 12 monosyllables), the ISI was 763 msec. $^{2}$ The experiment lasted $45 \mathrm{~min}$.

\section{Results and Discussion}

Number of transformations. Mean number of transformations in the four conditions is shown in Table 3. A two-way repeated measures ANOVA yielded a significant effect of syllable number $\left[F(1,30)=6.25, M S_{\mathrm{e}}=414.65\right]$, with one-syllable stimuli yielding an average of 9.4 more transformations than did two-syllable stimuli. The effect of lexical status was not reliable $\left[F(1,30)<1, M S_{\mathrm{e}}=\right.$ 420.94], nor was the interaction of the two variables reliable $\left[F(1,30)<1, M S_{\mathrm{e}}=467.57\right]$.

Number of forms. As can be seen in Table 3, the form analyses revealed small, although consistent, differences across words and pseudowords. Statistical analyses yielded no significant effect of lexicality $\left[F(1,30)=1.69, M S_{\mathrm{e}}=\right.$ $6.91]$ or syllable number $\left[F(1,30)<1, M S_{\mathrm{e}}=12.60\right]$ and no interaction between the two $\left[F(1,30)<2, M S_{\mathrm{e}}=\right.$ 17.76]. Informal item analyses showed similarly equivocal findings. Comparisons across word-pseudoword pairs (e.g., skunk-swunk; fender-derfen) on the number of transformations reported showed no consistent lexical differences.

Across the two global measures of the verbal transformation effect, the results were comparable to those of Experiment 1 , yielding no clear differences between words and pseudowords. One unexpectedfinding was that pseudowords failed to yield more forms than did words, as was found in Experiment 1. Another surprise was that mono- syllables produced more transformations than did bisyllables. Reasons for these outcomes will be discussed shortly.

Form subanalysis. The results of the form subanalysis (shown in Table 4) told a much more interesting story. Focusing first on the monosyllables, a comparison with the data in Experiment 1 (Table 2) revealed a very close replication. Veridical transformations were reported more often when the stimulus was a word than when it was a legal pseudoword $\left[F(1,30)=18.57, M S_{\mathrm{e}}=0.02\right]$. The magnitude of the effect was identical across experiments (.14). When nonveridical transformations were reported, differences emerged in the frequency of pseudoword reports, not of word reports, just as in Experiment 1: Word transformations ranged from .20 to .25. Pseudoword transformations were reliably more frequent when the stimulus was itself a pseudoword rather than a word $[F(1,30)=$ $18.43, M S_{\mathrm{e}}=0.03$ ].

The asymmetry in the frequency with which the veridical stimulus was reported was more pronounced with the bisyllables, for which a .27 difference between words and pseudowords was found $\left[F(1,30)=34.16, M S_{\mathrm{e}}=0.003\right]$. This difference was reliably greater than that found with monosyllables $\left[F(1,30)=5.96, M S_{\mathrm{e}}=0.02\right]$. Reports of nonveridical transformations for the bisyllables yielded a pattern of results opposite to that for the monosyllables. Instead of nonveridical word transformations' being equally frequent for words and pseudowords, there were more than twice as many nonveridical word reports for pseudowords than for words. Both types of stimuli yielded nearly identical proportions of nonveridical pseudoword transformations.

Except for this one anomalous outcome, the form subanalysis replicated the results of Experiment 1 and generalized the findings to another type of stimuli, bisyllables. We hypothesized that the source of this anomaly, and possibly of the main effect of syllable number in the numberof-transformations analysis, could be the cross-splicing procedure used to create the bisyllabic pseudowords. Reordering the syllables did not affect the intelligibilityof the stimuli; however, it did cause the intonation of some of the stimuli to sound slightly unnatural. More important, the ISI might not have been sufficiently long to prevent the perceptual reordering of the syllables in the recycling sequences back to form the word (derfen $\rightarrow$ fender). Inspec-

Table 4

Mean Proportions of Veridical and Nonveridical Transformations Reported as a Function of Stimulus Type in Experiment 2 With Cross-Spliced Stimuli (With Mean Numbers of Transformations)

\begin{tabular}{|c|c|c|c|c|c|c|c|c|}
\hline \multirow[b]{4}{*}{ Transformation Type } & \multicolumn{8}{|c|}{ Stimulus Type } \\
\hline & \multicolumn{4}{|c|}{ One-Syllable } & \multicolumn{4}{|c|}{ Two-Syllable } \\
\hline & \multicolumn{2}{|c|}{ Word } & \multicolumn{2}{|c|}{ Pseudoword } & \multicolumn{2}{|c|}{ Word } & \multicolumn{2}{|c|}{ Pseudoword } \\
\hline & Prop. & No. & Prop. & No. & Prop. & No. & Prop. & No. \\
\hline Veridical transformations & .42 & 24.03 & .28 & 16.32 & .42 & 19.55 & .15 & 9.71 \\
\hline Nonveridical transformations & .58 & 33.23 & .72 & 42.19 & .58 & 31.64 & .85 & 36.58 \\
\hline Words & .23 & 9.48 & .20 & 8.10 & .17 & 8.52 & .41 & 18.42 \\
\hline Pseudowords & .35 & 23.74 & .52 & 34.10 & .41 & 23.10 & .44 & 18.16 \\
\hline
\end{tabular}

Note-Standard deviations ranged from .12 to .29 . 
Table 5

Mean Numbers of Transformations and Forms Reported as a Function of Lexical Status and Number of Syllables (With Standard Deviations) in Experiment 2 With Unspliced Stimuli

\begin{tabular}{lccccc}
\hline & \multicolumn{2}{c}{ Transformations } & & \multicolumn{2}{c}{ Forms } \\
\cline { 2 - 3 } \cline { 5 - 6 } Stimulus Type & $M$ & $S D$ & & $M$ & $S D$ \\
\hline One-Syllable & & & & \\
$\quad$ Word & 52.90 & 29.18 & & 7.67 & 3.89 \\
$\quad$ Pseudoword & 59.50 & 32.60 & 11.00 & 5.84 \\
Two-Syllable & & & & \\
$\quad$ Word & 55.10 & 29.80 & 8.53 & 5.87 \\
Pseudoword & 53.20 & 35.30 & 11.03 & 7.03 \\
\hline
\end{tabular}

tion of the forms that the listeners reported in this condition confirmed this suspicion. Of the transformations the listeners reported when hearing the bisyllabic pseudowords, syllable reorderings into their word counterparts (e.g., derfen perceived as fender) occurred $24 \%$ of the time. The syllables of bisyllabic words underwent such a reordering into their pseudoword counterparts less than $1 \%$ the time. The strong tendency to reorder the syllables of the bisyllabic pseudowords and to hear them as their word counterparts may account for the bias to report such a high proportion of nonveridical word transformations. In essence, the large proportion of nonveridical word reports (.41) was traded off for a drop in nonveridical pseudoword reports (.44). To test the accuracy of this account, we reran Experiment 2 with unspliced bisyllabic stimuli, rather than with spliced tokens.

\section{EXPERIMENT 3}

\section{Method}

Participants. Thirty participants from the same populations as those in Experiment 2 served in the experiment.

Procedure. The experiment was identical to Experiment 2, except that the unspliced tokens of the bisyllabic stimuli were used, instead of the spliced tokens.

\section{Results and Discussion}

The data from the two global measures of the verbal transformation effect, number of transformations and number of forms, are shown in Table 5. With unspliced tokens, there was no main effect of syllable number $[F(1,29)<1$, $\left.M S_{\mathrm{e}}=307.0\right]$ or of lexical status $\left[F(1,29)<1, M S_{\mathrm{e}}=\right.$
281.5] on the number of transformations reported. The only statistically reliable finding was in the number of forms reported, with pseudowords producing, on average, 2.92 more forms than did words $\left[F(1,29)=11.51, M S_{\mathrm{e}}=\right.$ 22.20]. Pseudowords produced more forms than did words for the bisyllables, as well as for the monosyllables, as had been found in Experiment 1 (Table 1).

The data from the form subanaly sis are shown in Table 6 . Cell by cell, the results closely resemble those in Experiment 2 (Table 4), except for the frequency with which nonveridical transformations were reported for bisyllabic pseudowords. With the unspliced stimuli, word transformations dropped from .41 to .17 , and pseudoword transformations rose from .44 to .65 . An examination of the forms listeners reported for the pseudowords showed that only $8 \%$ of the transformations reported were reorderings of the two syllables back into the original words, a sizable drop from that reported with the spliced stimuli (24\%).

The results of Experiment 3 indicate that reordering the syllables in Experiment 2 had a very localized effect on what listeners perceived, exaggerating reports of resegmentations. In all other respects, the conclusions are the same as those of Experiment 1: Pseudowords yield more forms than do words, which occurs because recycling words transform back into the veridical percept more often than do recycling pseudowords. This persistent lexical effect, which emerged yet again in the global form analysis and in the form subanalyses, gives further credence to the proposal that node stability is a critical factor in the verbal transformation effect.

\section{GENERAL DISCUSSION}

The aim of this study was to explore whether node stability, as put forth by node structure theory, provides a reasonable account of lexical effects in the verbal transformation effect. Although the exact predictions of node structure theory were not confirmed, the core idea underlying the cause of the verbal transformation effect, node stability itself, garnered some support.

One global measure of the verbal transformation effect (number of transformations) proved relatively insensitive to variation in lexical status, yielding only minor or inconsistent differences between words, legal pseudowords, and

Table 6

Mean Proportions of Veridical and Nonveridical Transformations Reported as a Function of Stimulus Type in Experiment 2 With Unspliced Stimuli (With Mean Numbers of Transformations)

\begin{tabular}{|c|c|c|c|c|c|c|c|c|}
\hline \multirow[b]{4}{*}{ Transformation Type } & \multicolumn{8}{|c|}{ Stimulus Type } \\
\hline & \multicolumn{4}{|c|}{ One-Syllable } & \multicolumn{4}{|c|}{ Two-Syllable } \\
\hline & \multicolumn{2}{|c|}{ Word } & \multicolumn{2}{|c|}{ Pseudoword } & \multicolumn{2}{|c|}{ Word } & \multicolumn{2}{|c|}{ Pseudoword } \\
\hline & Prop. & No. & Prop. & No. & Prop. & No. & Prop. & No. \\
\hline Veridical transformations & .39 & 21.70 & .25 & 14.73 & .41 & 21.90 & .18 & 7.50 \\
\hline Nonveridical transformations & .61 & 31.27 & .75 & 44.77 & .59 & 32.83 & .82 & 46.13 \\
\hline Words & .18 & 7.90 & .23 & 12.87 & .09 & 6.03 & .17 & 9.47 \\
\hline Pseudowords & .43 & 23.37 & .52 & 31.23 & .50 & 26.80 & .65 & 36.66 \\
\hline
\end{tabular}

Note-Standard deviations ranged from .13 to .26 . 
illegal pseudowords. In contrast, in Experiments 1 and 3, pseudowords produced more forms than did words in the overall form analysis, replicating Natsoulas (1965) and partially supporting the predictions of node structure theory. Although there was a trend in this same direction in both the monosyllabic and the bisyllabic data of Experiment 2, it was not large enough to reach statistical significance. We are inclined to believe that this smaller effect was a result of sampling variation, because the monosyllabic conditions were identical across Experiments 2 and 3. All that varied were the participants.

The form subanalyses took the lexical effect one step further and provided an explanation for why the effect was found. In doing so, the primary evidence in support of node stability was obtained. Words transformed into the veridical stimulus more often than did legal pseudowords. This outcome was found in all three experiments and generalized to two-syllable utterances. A comparison of the data across Tables 2, 4, and 6 shows that the findings are impressively consistent.

One puzzling outcome for which we do not as yet have an explanation is why monosyllables yielded such a smaller number of transformations and unique forms in Experiment 1 than in Experiments 2 and 3. The only change in methodology was in the ISI, with a much shorter interval in Experiment 1. Why this change should result in a decrease, rather than an increase, in reports is unclear. Whatever the cause, it is important to note that any effect of ISI variation was independent of lexical status. The types of transformations and their frequency increased in Experiments 2 and 3, as compared with Experiment 1, but the effects of word-likeness did not change in magnitude. Inspection of the results from the form subanalyses across experiments shows that the proportions in corresponding conditions remained fairly constant.

The type of node stability identified in the present data is slightly different from that proposed by MacKay et al. (1993). On the basis of their definition, stability refers to the frequency of transformation: The more stable a node is, the less likely it is that it will undergo transformation. Stability is a function of satiation, with more stable representations (e.g., words vs. pseudowords) being satiated less often. Instead, our data show that it was not the frequency of transformation that differed between words and pseudowords; rather, it was the likelihood of the stimulus's transforming back into its veridical form that differed across conditions. Node stability is tied to the recovery mechanism, which the present experiments have demonstrated depends on two properties of the stimulus, its lexical status and its phonological permissibility. Recovery is greater for words than for pseudowords and is greater for phonologically legal utterances than for illegal utterances.

The discussion so far has focused solely on one theory, node structure theory, because the concept of node stability emerges from the operation of its inner workings. It is also useful to consider how other models of word perception compare with node structure theory in accounting for the data. Models such as Merge (Norris et al., 2000), TRACE (McClelland \& Elman, 1986), and NAM (Luce
\& Pisoni, 1998; Vitevitch \& Luce, 1999) are structurally similar to node structure theory, in that there are hierarchically arranged levels of representation. Such an organization lends itself to explaining lexicality effects; pseudoword processing is assumed to occur sublexically, and word processing lexically. Another important feature that these models share with node structure theory is that their network architectures consist of excitatory and inhibitory connections among representational (e.g., lexical) units, providing a means for competition among representations. The similarities in their overall designs to node structure theory suggests that many of the features are in place to account for the present results.

Where node structure theory differs from these models is in some of the details of how representational units function, and these are necessary to explain why transformations are perceived. Most notably, those models have no satiationlike process, and something like it is probably necessary to explain transformation perception. In their present instantiations, the stimulus would be perceived veridically every time it was presented, yet the existence of the illusion itself demonstrates that this is not how humans perceive. Of course, there are no barriers to incorporating such a mechanism into these three models or others like them.

To fully account for the present results, more detail would also have to be provided, in some models, on how pseudowords are perceived and how they compete for recognition, since pseudowords constitute a large number of transformations and give rise to a large number of transformations. In node structure theory, pseudowords are perceived by constructing temporary lexical representations on the fly from a combination of sublexical units. The same could be done in models like Merge and TRACE as well. Thus, the present data favor node structure theory only to the extent that it has all the processes in place to explain the results. Precisely because of this, the model provides a useful and ready framework for investigating node stability in the context of the verbal transformation effect.

The results of this investigation also have broader implications. This study builds on that of MacKay et al. (1993) in showing that the verbal transformation effect can be harnessed so we can learn about the processes underlying word perception. The recycling word methodology provides a unique window into the processing system. In the present case, it shows that representational units must satiate, in addition to activating and deactivating. At the same time, the lexicality effects found across the three experiments demonstrate that the task taps processes found with more traditional paradigms (e.g., lexical decision or phoneme classification) and provide converging evidence that demonstrates the role of lexical memory in perception. What was surprising was the form of the lexical effect. Lexical memory did not merely bias the listener to hear a word, as opposed to a pseudoword, as is generally found. Rather, it also helped stabilize perception of the veridical stimulus so that fewer nonveridical transformations were heard. How this process comes about is the next question to be explored in this project. 
In closing, it is worthwhile to consider what the present data tell us about the measures of the verbal transformation effect. The form subanalysis proved to be a richer source of information about lexical influences in the verbal transformation effect than were the two global measures. The global measures may be best for revealing the experimental conditions necessary to produce the verbal transformation effect, whereas the finer grained analyses may provide the greatest insight into the operation of the processes underlying the phenomenon and, thus, word perception itself.

\section{REFERENCES}

Luce, P. A., \& Pisoni, D. B. (1998). Recognizing spoken words: The neighborhood activation model. Ear \& Hearing, 19, 1-36.

Luce, P. A., Pisoni, D. B., \& Goldinger, S. D. (1990). Similarity neighborhoods of spoken words. In G. T. M. Altmann (Ed.), Cognitive models of speech processing (pp. 148-172). Cambridge, MA: MIT Press.

MAcKAY, D. G. (1987). The asymmetrical relationship between speech perception and production. In H. Heuer \& A. F. Sanders (Eds.), Perspectives on perception and action (pp. 301-333). Hillsdale, NJ: Erlbaum.

MacKay, D. G., Burke, D. M., \& Stewart, R. (1998).H.M.'s language production deficits: Implications for relations between memory, semantic binding, and the hippocampal system. Journal of Memory \& Language, 38, 28-69.

MacKay, D. G., Wulf, G., Yin, C., \& Abrams, L. (1993). Relations between word perception and production: New theory and data on the verbal transformation effect. Journal of Memory \& Language, 32, 624-646.

McClelland, J. L., \& Elman, J. L. (1986). The TRACE model of speech perception. Cognitive Psychology, 18, 1-86.

Natsoulas, T. (1965). A study of the verbal-transformation effect. American Journal of Psychology, 78, 257-263.

Norris, D., McQueen, J., \& CUTLER, A. (2000). Merging information in speech recognition: Feedback is never necessary. Behavioral \& Brain Sciences, 23, 299-370.

Vitevitch, M. S., \& Luce, P. A. (1999). Probabilistic phonotactics and neighborhood activation in spoken word recognition. Journal of Memory \& Language, $\mathbf{4 0}, 374-408$.

WARREN, R. M. (1961a). Illusory changes in repeated words: Differ- ences between young adults and the aged. American Journal of Psychology, 74, 506-516.

WARREN, R. M. (1961b). Illusory changes of distinct speech upon repetition: The verbal transformation effect. British Journal of Psychology, 52, 249-258.

WARREN, R. M. (1983). Auditory illusions and their relation to mechanisms normally enhancing accuracy of perception. Journal of the Audiological Engineering Society, 31, 623-628.

WARREN, R. M., \& GREGORY, R. L. (1958). An auditory analogue of the visual reversible figure. American Journal of Psychology, 71, 612-613.

WARREN, R. M., \& WARREN, R. P. (1966). A comparison of speech perception in childhood, maturity, and old age by means of the verbal transformation effect. Journal of Verbal Learning \& Verbal Behavior, $\mathbf{5}, 142-146$

YIN, C., \& MACKAY, D. G. (1992, April). Auditory illusions and aging: Transmission of priming in the verbal transformation paradigm. Poster presented at the Cognitive Aging Conference, Atlanta.

\section{NOTES}

1. One additional test of node stability in node structure theory that we undertookinvolved measuring the stretch of time (i.e., the number of uninterrupted cycles) that the veridical forms of the three types of stimuli were heard. An analysis of 5 of the participants' data showed that words did not yield longer runs of hearing the veridical stimulus than did pseudowords (legal or illegal). Node structure theory predicts such a difference because word representations should be more stable and, thus, more immune to transformation than nonword representations.

2. A software problem caused the unexpected variation in ISI, which was not discovered until after the experiment had been run. It is highly unlikely that this small variation in ISI affected the results in any meaningful way, since the results of Experiment 1 were replicated with this same variation in ISI occurring across stimuli (these data are available from the authors upon request). Also, Experiment 2 could not be run at the short ISI of Experiment 1 without causing the listeners to frequently segment the two syllables of the spliced pseudowords (e.g., derfen) and perceptually group the second syllable with the first syllable of the following presentation of the pseudoword to form the word from which the pseudoword was created (e.g., derfen-derfen $\rightarrow$ der-fender-fen). A longer ISI was therefore necessary. Inclusion of the monosyllables in the experimental design allowed us to assess whether and how this slowdown affected performance by comparing the monosyllabic data across Experiments 1 and 2. Comparison of the data suggests that it had no measurable effect.

\section{APPENDIX}

Stimuli Used in Experiments 1-3

\begin{tabular}{lll}
\hline Words & $\begin{array}{c}\text { Legal } \\
\text { Pseudowords }\end{array}$ & $\begin{array}{c}\text { Illegal } \\
\text { Pseudowords } \\
\text { (Experiment 1 Only) }\end{array}$ \\
\hline skunk & \multicolumn{2}{c}{ One-Syllable } \\
sketch & swunk & srunk \\
skin & sletch & sretch \\
bliss & driss & dliss \\
blond & trond & tlond \\
grave & trave & \\
& \multicolumn{1}{c}{ Two-Syllable } \\
center & tercen & \\
fender & derfen & \\
signal & nalsig & \\
stencil & cilsten & \\
cosmos & moscos & \\
fluster & terflus & \\
\hline
\end{tabular}

(Manuscript received September 22, 1999;

revision accepted for publication November 26, 2001.) 